\title{
Temporal evaluation of computed tomographic scans at a Level 1 trauma department in a central South African hospital
}

\begin{tabular}{|c|c|}
\hline \multicolumn{2}{|c|}{$\begin{array}{l}\text { Authors: } \\
\text { Tony Tiemesmann }{ }^{1} \\
\text { Jacques Raubenheimer }{ }^{2} \\
\text { Coert de Vries }{ }^{1}\end{array}$} \\
\hline \multicolumn{2}{|c|}{$\begin{array}{l}\text { Affiliations: } \\
{ }^{1} \text { Department of Clinical } \\
\text { Imaging Sciences, School of } \\
\text { Medicine, Faculty of Health } \\
\text { Sciences, Pelonomi Hospital } \\
\text { and University of the Free } \\
\text { State, Bloemfontein, South } \\
\text { Africa }\end{array}$} \\
\hline $\begin{array}{l}{ }^{2} \text { Department } \\
\text { Faculty of Hea } \\
\text { University of } \mathrm{t} \\
\text { Bloemfontein, }\end{array}$ & $\begin{array}{l}\text { Biostatistics, } \\
\text { th Sciences, } \\
\text { he Free State, } \\
\text { South Africa }\end{array}$ \\
\hline \multicolumn{2}{|c|}{$\begin{array}{l}\text { Corresponding author and } \\
\text { email: } \\
\text { Tony Tiemesmann } \\
\text { tonytiemesmann@vodamail. } \\
\text { co.za }\end{array}$} \\
\hline \multicolumn{2}{|c|}{$\begin{array}{l}\text { Dates: } \\
\text { Received: } 06 \text { May } 2015 \\
\text { Accepted: } 06 \text { Oct. } 2015 \\
\text { Published: } 18 \text { Mar. } 2016\end{array}$} \\
\hline \multicolumn{2}{|c|}{$\begin{array}{l}\text { How to cite this article: } \\
\text { Tiemesmann T., } \\
\text { Raubenheimer J., \& } \\
\text { de Vries C. Temporal } \\
\text { evaluation of computed } \\
\text { tomographic scans at a Level } \\
1 \text { trauma department in a } \\
\text { central South African } \\
\text { hospital. S Afr J Rad. } \\
2016 ; 20(1) ; \text { Art. \#836, } \\
5 \text { pages. http://dx.doi. } \\
\text { org/10.4102/sajr.v20i1.836 }\end{array}$} \\
\hline \multicolumn{2}{|c|}{$\begin{array}{l}\text { Copyright: } \\
\text { (c) 2016. The Authors. } \\
\text { Licensee: AOSIS. This w } \\
\text { licensed under the Crea } \\
\text { Commons Attribution } \\
\text { License. }\end{array}$} \\
\hline \multicolumn{2}{|l|}{ Read online: } \\
\hline 口itpr & $\begin{array}{l}\text { Scan this QR } \\
\text { code with your } \\
\text { smart phone or } \\
\text { mobile device } \\
\text { to read online. }\end{array}$ \\
\hline
\end{tabular}

Background: Time is a precious commodity, especially in the trauma setting, which requires continuous evaluation to ensure streamlined service delivery, quality patient care and employee efficiency.

Objectives: The present study analyses the authors' institution's multi-detector computed tomography (MDCT) scan process as part of the imaging turnaround time of trauma patients. It is intended to serve as a baseline for the institution, to offer a comparison with institutions worldwide and to improve service delivery.

Method: Relevant categorical data were collected from the trauma patient register and radiological information system (RIS) from 01 February 2013 to 31 January 2014. A population of 1107 trauma patients who received a MDCT scan was included in the study. Temporal data were analysed as a continuum with reference to triage priority, time of day, type of CT scan and admission status.

Results: The median trauma arrival to MDCT scan time (TTS) and reporting turnaround time (RTAT) were 69 (39-126) and 86 (53-146) minutes respectively. TTS was subdivided into the time when the patient arrived at trauma to the radiology referral (TTRef) and submission of the radiology request, to the arrival at the MDCT (RefTS) location. TTRef was statistically significantly longer than RefTS $(p<0.0001)$. RTAT was subdivided into the arrival at the MDCT to the start of the radiology report (STR) and time taken to complete the report (RT). STR was statistically significantly longer than RT $(p<0.0001)$.

Conclusion: The time to scan (TTS) was comparable to, but unfortunately the report turnaround time (RTAT) lagged behind, the findings of some first-world institutions.

\section{Introduction}

Productivity, cost, quality and time are the main concerns in every workplace. Improved productivity can be achieved by addressing all these factors. In the trauma setting, estimating how much time a resource requires for each step in the process of patient care is necessary to improve efficiency and workflow. ${ }^{1}$ The 60-minute timeframe between injury and definitive treatment is commonly referred to as the golden hour, but it is an arbitrary measurement. Each trauma patient should be treated individually and as quickly as possible, depending on the extent of the injury. ${ }^{2}$

It has become standard practice to rely on multi-detector computed tomography (MDCT) scanning in the early diagnosis of injury in the trauma patient. ${ }^{3}$ Many factors influence the traumaradiology timeline of the injured patient. Different trauma treatment protocols are used around the world, including custom-designed systems for the specific trauma department and advanced trauma life support (ATLS). ATLS focuses on a specific sequence of procedures and interventions to be performed in dealing with an injured patient. ${ }^{4}$ Several studies have shown that a CT scanner located within the trauma department can reduce the time taken to obtain a definite diagnosis. ${ }^{5}$ An emergency MDCT scan requires expert evaluation by a qualified radiologist. It is through an approved radiology report that the radiologist adds the most value to patient care. ${ }^{6}$

In the present study, we evaluated the trauma-radiology timeline in a non-trauma departmentlocated MDCT scanner with the use of ATLS protocols to establish an institutional baseline and improve productivity and patient care. To our knowledge, no such study has previously been performed in Africa. 


\section{Research method and design Setting}

Pelonomi Tertiary Hospital is a 720-bed facility and offers the only Level 1 trauma unit in central South Africa, according to the Trauma Society of South Africa (TSSA). ${ }^{7}$ Registrars in a surgical discipline are required to do a three-month rotation in trauma. All doctors have current ATLS accreditation. An institution-specific pre-hospital-based triage system is used at the institution (Table 1).

The Department of Clinical Imaging Sciences is in a building adjacent to the trauma department and is connected via a passageway of $50 \mathrm{~m}$. Two CT scanners are on site in the radiology department: a single-slice GE (General Electric, Milwaukee, USA) HiSpeed and a 64-slice GE LightSpeed Discovery (General Electric, Milwaukee, USA). The 64-slice unit is the preferred scanner for trauma and elective CT scans. The single-slice unit is only used as a backup if the other unit fails.

The Department of Clinical Imaging Sciences is fully digital, and all diagnostic imaging is sent to an AGFA (AGFA HealthCare, Mortsel, Belgium) radiology information system (RIS). Reporting is done via AGFA (AGFA HealthCare, Mortsel, Belgium) voice-recognition software.

\section{Design}

A retrospective cohort study was conducted over a 12-month period at Pelonomi Hospital. The data were collected from 01 February 2013 up to and including 31 January 2014. All patients referred for a MDCT scan from the trauma unit during their initial visit were included in the study.

TABLE 1: Triage coding.

\begin{tabular}{lll}
\hline Priority coding & Colour coding & Urgency \\
\hline P1 & Red & Life threatening \\
P2 & Yellow & Potentially life threatening \\
P3 & Green & Walking \\
P4 & Black & Not salvageable/dead \\
\hline
\end{tabular}

\section{Procedure}

The time of patient arrival at the trauma department was collected from the trauma register. The time when the scan was requested, the starting time of the MDCT scan and the times when the radiology report was initiated and completed were collected from the RIS. All radiology reports were reported and finalised by the registrar on duty. By subtracting the appropriate times of day, the time of interest was calculated. A trauma-radiology timeline is shown in Figure 1.

Various patient demographic factors were recorded, including age, sex, trauma triage priority, type of MDCT scan, admission and referral status and trauma deaths after MDCT scan.

A 'shift change-over' time analysis was also included of the two-hour time period between 6 and 8 o' clock in the mornings and evenings. This is the period when nurses, radiographers and porters change shift. Registrar handover rounds and other morning meetings are also held during this time.

MDCT studies were captured as either a pan scan that involved three or more body areas or a regional scan that involved one anatomical compartment (e.g., the abdomen).

\section{Statistical analyses}

The analysed data were reported by means of frequencies and percentages and continuous data using ranges, medians and means with standard deviations. Confidence intervals of 95\% were also included.

Time differences for some categorical variables (e.g., trauma priority) were computed by means of analysis of statistical variance (ANOVA) or independent $t$-tests. Different times in the whole process were compared by means of paired $t$-tests.

\section{Ethical considerations}

The data were gathered retrospectively after the necessary permissions had been obtained. Patient information was kept

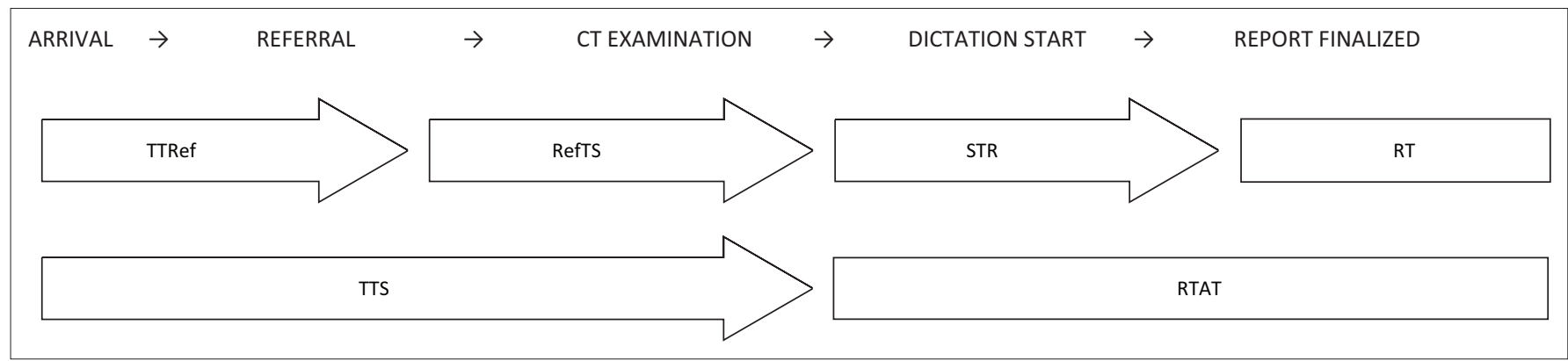

FIGURE 1: The trauma-radiology timeline.

TTRef - time to referral: Time from when the patient arrived in trauma until the time that radiology referral was requested on the hospital information system (HIS, Meditech, South Africa).

RefTS - referral to scan: Time from when the radiology referral was requested on Meditech until the time that the patient arrived at the CT scanner.

STR - scan to report: Time from when the patient arrived at the CT scanner until the time that dictation was started on the radiology information system (RIS).

RT - reporting time: Time from when the dictation was started on the RIS until the time that the report was finalised.

TTS - time to scan: TTRef added to RefTS.

RTAT - report turnaround time: STR added to RT. 
anonymous, so that none of the identifying information gathered for the data analysis was used in this study.

\section{Results}

A total of 4977 patients were seen in the trauma department, 1144 of whom underwent a MDCT scan. Thirty-seven patients were excluded from the study owing to incomplete entered data and/or illegible handwriting in the trauma unit register and/or data mismatch between the trauma unit register entry and data on the RIS. Of 1107 patients included in the study, 905 patients (82\%) were male, and the sample had a median age of 30 (interquartile range (IQR) 23-39) years. Most (62\%) patients were triaged as priority 2 (Table 1 ). In all, 734 patients were admitted, 646 to a normal ward and 88 to the intensive care unit (ICU). As expected, most patients admitted to ICU were triaged as priority $1(74 \%)$. Seventythree $(6.6 \%)$ patients were transferred to other local tertiary hospitals and one $(0.01 \%)$ patient signed a refusal-of-hospitaltreatment (RHT). The overall mortality rate for patients who underwent a MDCT scan but died shortly thereafter in trauma was $3.9 \%$ (Table 2).

Median time until start of the MDCT scan (TTS) was 69 minutes (IQR 39-126) with time-to-referral of 38 minutes (IQR 18-78) taking significantly $(t=11.5, d f=1106, p<0.0001)$ longer than RefTS at 26 minutes (IQR 14-44). An ANOVA with ad hoc Scheffe tests on the TTS times across the four shift times showed that there was a statistically significant difference $(F=12.9, d f=3, p<0.0001)$ in TTS between the morning 'change-over' time period and the other three shifts (day shift, night shift and the evening 'change-over' period), although there were no differences between these three themselves (Figure 2). There was no statistically significant difference in TTS compared with the patient triage priority. Median TTS of pan scan patients was 98 minutes (IQR 55.5156.5) with a TTR of 49.5 minutes (IQR 31.5-102.5) and RefTS of 30 minutes (IQR 17-54.5). This was statistically significantly longer $(t=2.7, d f=1105, p=0.0072)$ than for regional scan patients, with a median of 65 minutes (IQR 38-123) (Figure 3).

TABLE 2: Patient demographics.

\begin{tabular}{lcc}
\hline Patient and admission details & $\mathbf{f}$ & $\mathbf{\%}$ \\
\hline Sex & 202 & 18 \\
Female & 905 & 82 \\
Male & & \\
Trauma triage priority & 225 & 20 \\
Priority 1 & 685 & 62 \\
Priority 2 & 197 & 18 \\
Priority 3 & & \\
Type of scan & 132 & 12 \\
Pan scan & 975 & 88 \\
Regional scan & & \\
Admission status & 256 & 23 \\
Discharged & 88 & 8 \\
Admitted to ICU & 73 & 7 \\
Transferred to another hospital & 646 & 58 \\
Admitted to a normal ward & 43 & 4 \\
Died in trauma after CT scan & 1 & $<1$ \\
Refusal of hospital treatment & & \\
\hline
\end{tabular}

$f$, frequency.
Median report turnaround time (RTAT) was 86 minutes (IQR 53-146). As with TTS, an ANOVA showed a statistically significant difference $(F=12.4, d f=3, p<0.0001)$ in RTAT between the morning 'change-over' time period and the remaining three shifts, but again no difference between the other three shifts themselves. RTAT was longer in P1 and pan scan patients, but it was only statistically significant in the latter $(t=4.5, d f=1105, p<0.0001)$ (Figure 4). As seen in Figure 1, the RTAT consists of STR and RT. STR (with a median of 48 minutes and an IQR of 30-77 minutes) was significantly $(t=26.5, d f=1106, p<0.0001)$ longer than RT (which had a median of only 12 minutes and an IQR of 7-20 minutes). It is also evident that the administrative process preceding a scan took slightly longer (but was still statistically significant: $t=5.3, d f=1106, p<0.0001)$ than the actual scanning process, with TTS having a median and mean of 69 and 97 minutes respectively, compared with 65 and 81 minutes for RTAT.

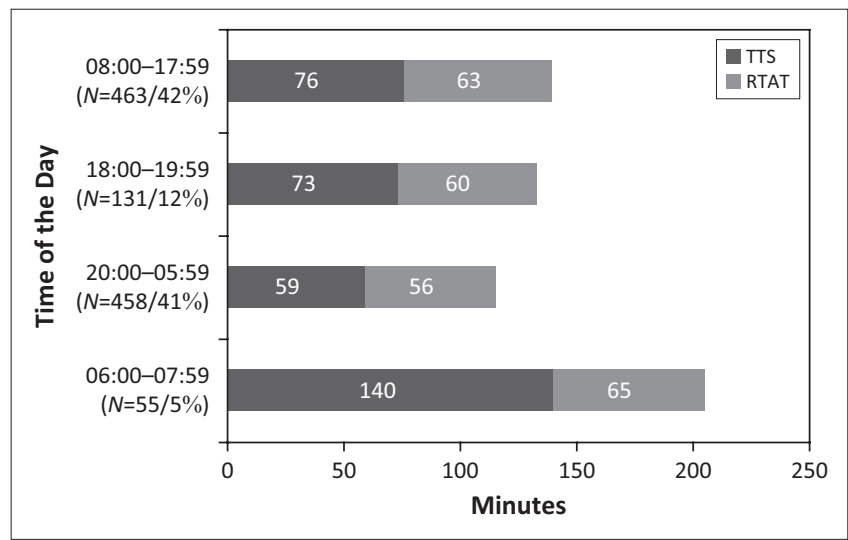

FIGURE 2: Time to scan (TTS) and report turnaround time (RTAT) during different work-hour shifts including number of patients $(N)$ and percentage of the total.

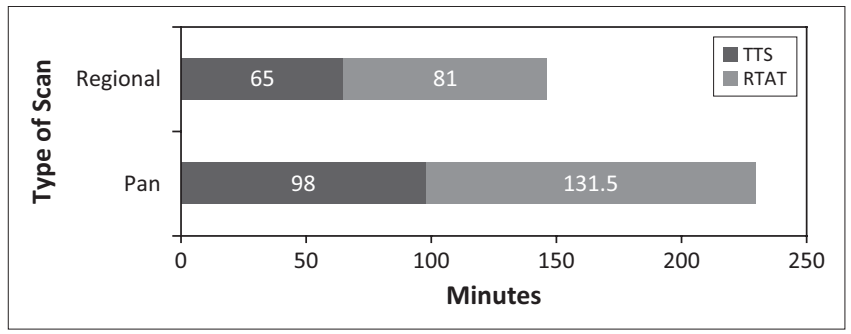

FIGURE 3: Time to scan (TTS) and report turnaround time (RTAT) in regional and pan scan patients.

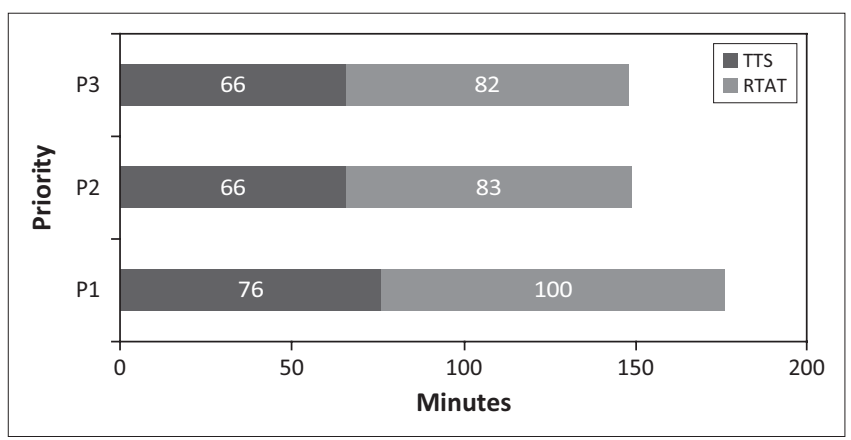

FIGURE 4: Time to scan (TTS) and report turnaround time (RTAT) compared with the triage priority of the patient. 


\section{Discussion}

As South Africa embarks on health reform in the shape of national health insurance (NHI), data from the current health system need to be evaluated to determine where challenges might lie and to address them in a timely fashion. ${ }^{8}$ An analysis by the National Injury Mortality Surveillance System (NIMSS) found that, of all non-natural deaths in South Africa, violence and transport-related injuries were the leading causes of death. ${ }^{9}$

The main aim of the present retrospective study was to evaluate time as a resource in MDCT scanning at a public Level 1 trauma unit. Although demographic factors and comparisons were also apparent, only two of the factors identified can be compared to other publications: TTS and RTAT (Table 3).

TTSs comparable to our results were obtained by Easton et al. ${ }^{10}$ in Australia, where the MDCT scanner is also located outside the trauma unit, and Fung Kon Yin et al., ${ }^{3}$ where a dedicated trauma MDCT scanner was employed but with the ATLS trauma algorithm. In Canada, Rados etal., ${ }^{11}$ where a customised trauma protocol with the MDCT outside the trauma unit was employed, compared different types of trauma team responses. The most astonishing TTS of 8 minutes $(\mathrm{SD}=5.7)$ was reported by Hillbert et al. ${ }^{12}$ in Germany, where a dedicated trauma MDCT and accompanying protocol are used. Lee et al. ${ }^{5}$ compared before-and-after scenarios of placing the MDCT scanner in the trauma unit with a non-ATLS protocol in Hong Kong. They found a similar mean TTS to ours of 102 minutes after introduction of the MDCT scanner in the trauma unit. Wurmb et al..$^{13}$ demonstrated a significant effect on TTS when the location of the MDCT was changed to the trauma unit with a necessarily altered trauma protocol. Moving the MDCT scanner closer to the trauma unit will improve RefTS, and thus TTS, by approximately 5 minutes, but this is not realistic in our setting because $85 \%$ of MDCT scans are not referred from trauma.
Weinberg et al. ${ }^{14}$ also evaluated the RTAT of radiology residents in trauma patients. Boland et al. ${ }^{15}$ proved that clinician incentives can reduce RTAT by almost 9 minutes in a pay-for-performance (PFP) programme. STR is the main contributor to our long RTAT because the MDCT scanning time is included. Owing to data limitations, our RTAT includes patient transfer to the MDCT scan bed and MDCT scanning time. Other studies calculate RTAT as from completion of the scan till the finalised radiology report. ${ }^{13,14}$ It is probably because of this fact that our median RTAT compares poorly to other studies. As per departmental protocol, an initial report is always phoned to the referring call doctor. Registrars are the only radiology doctors doing trauma MDCT reporting at the hospital, with consultant back-up available on-site during office hours and via tele-radiology after hours. Asking for consultant assistance prior to starting a report on the system may also contribute to the prolonged RTAT. It should be noted that consultant advice is only requested when the registrar feels unsure of his/her diagnosis. Help with patient transfer back to the trauma unit owing to lack of staff, prior to the start of the report, is a common occurrence.

\section{Limitations of the study}

In conducting this retrospective study, it was found that the trauma register time entries were inconsistent.

\section{Recommendations}

The morning 'change-over' shift emerged as the most worrisome period and could be better managed by ensuring adequate staffing at all times. Future work may include prospective studies to identify even more specific areas of possible time delay and time management. Such areas could include a further breakdown of RefTS and STR with transfer times to and from trauma, primary survey imaging, and

TABLE 3: Comparison with previous studies.

\begin{tabular}{|c|c|c|c|c|c|c|}
\hline Study & Year & $\mathbf{N}$ & Time to scan (minutes) & Variability & Report turnaround time (minutes) & Variability \\
\hline Tiemesmann et al. & 2016 & 1107 & 69 (median), 98 (mean) & $I Q R=39-126, S D=88$ & 86 (median), 113.2 (mean) & $I Q R=53-146, S D=88$ \\
\hline \multirow[t]{2}{*}{ Rados et al. ${ }^{11}$} & \multirow[t]{2}{*}{2013} & 58 & 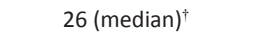 & $I Q R=19.5-36.5$ & \multirow[t]{2}{*}{ N/A } & \multirow[t]{2}{*}{$\mathrm{N} / \mathrm{A}$} \\
\hline & & 30 & 49 (median) & $\mathrm{IQR}=32-80.5$ & & \\
\hline Easton et al. ${ }^{10}$ & 2012 & 233 & 76 (median) & $\mathrm{IQR}=52-115$ & N/A & $\mathrm{N} / \mathrm{A}$ \\
\hline \multirow[t]{2}{*}{ Lee et al. ${ }^{5}$} & \multirow[t]{2}{*}{2009} & 111 & $197(\text { mean) })^{\S}$ & Not supplied & \multirow[t]{2}{*}{$\mathrm{N} / \mathrm{A}$} & \multirow[t]{2}{*}{$\mathrm{N} / \mathrm{A}$} \\
\hline & & 110 & $102($ mean) & Not supplied & & \\
\hline \multirow[t]{2}{*}{ Wurmb et al. ${ }^{13}$} & \multirow[t]{2}{*}{2009} & \multirow[t]{2}{*}{161} & 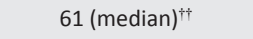 & $I Q R=20-32$ & \multirow[t]{2}{*}{ N/A } & \multirow[t]{2}{*}{ N/A } \\
\hline & & & 18 (median) & $\mathrm{IQR}=14-24$ & & \\
\hline Fung Kon Jin et al. ${ }^{3}$ & 2008 & 235 & 82 (median) & $\mathrm{IQR}=62-122$ & $\mathrm{~N} / \mathrm{A}$ & $\mathrm{N} / \mathrm{A}$ \\
\hline Hillbert et al. ${ }^{12}$ & 2007 & 139 & 8 (mean) & $S D=5.7$ & $\mathrm{~N} / \mathrm{A}$ & N/A \\
\hline Weinberg et al. ${ }^{14}$ & 2015 & 114440 & $\mathrm{~N} / \mathrm{A}$ & N/A & 40.2 (mean) & Not supplied \\
\hline \multirow[t]{2}{*}{ Boland et al. ${ }^{15}$} & \multirow[t]{2}{*}{2010} & 99959 & \multirow[t]{2}{*}{$\mathrm{N} / \mathrm{A}$} & \multirow[t]{2}{*}{ N/A } & $13.9(\text { mean })^{\S \S}$ & $S D=54.8$ \\
\hline & & 91379 & & & 5.2 (mean) $)^{4 \pi}$ & $S D=34.6$ \\
\hline
\end{tabular}

SD, standard deviation; N/A, not applicable; IQR, interquartile range.

$\dagger$ Full trauma team attending to the injured patient.

$\$$ Non-trauma team response to the injured patient.

$\S$ Multidetector computed tomography scanner (MDCT) located outside trauma department.

IMDCT located within trauma department.

†MDCT located within trauma department.

\$MDCT located outside trauma department.

$\S \S$ Before the introduction of pay-for-performance (PFP) incentives.

१ๆ After introduction of PFP incentives. 
various waiting times for patients and staff. A direct comparison is not meaningful because of many factors; for example, trauma protocol, MDCT scanner location and staff complement. It does, however, give an indication of our current level of service delivery.

\section{Acknowledgements}

\section{Competing interests}

The authors declare that they have no financial or personal relationships which may have inappropriately influenced them in writing this article.

\section{Authors' contributions}

C.S.d.V. (University of the Free State) was the study leader, T.N.T. (University of the Free State) was responsible for data sampling and writing the article, and J.R. (University of the Free State) made conceptual contributions and performed all the statistical analyses. A heartfelt thank you to Dr Elize Esterhuizen, and the Pelonomi radiographers and Trauma Department staff.

\section{References}

1. Kaplan RS, Porter ME. How to solve the cost crisis in health care. Harv Bus Rev. 2011;89:46-61.

2. Rogers FB. The golden hour in trauma: Dogma or medical folklore?. J Lanc Gen Hosp. 2014;9:11-13.
3. Fun Kon Jin PHP, Van Geene AR, Linnau KF, Jurkovich GJ, Ponsen KJ, Goslings JC. Time factors associated with CT scan usage in trauma patients. Euro J Rad. 2009;72:134-138. http://dx.doi.org/10.1016/j.ejrad.2008.06.022

4. American College of Surgeons Committee on Trauma. ATLS advanced trauma life support program for doctors. Chicago: American College of Surgeons; 2013.

5. Lee K, Graham C, Lam J, Yeung J, Ahuja A, Rainer T. Impact on trauma patient management of installing a computed tomography scanner in the emergency department. Injury. 2009;40:873-875. http://dx.doi.org/10.1016/j.injury.2008.12.001

6. Boland GWL, Guimaraes AS, Mueller PR. Radiologist report turnaround
Expectations and solutions. Eur Radiol. 2008;18:1326-1328. http://dx.doi. org/10.1007/s00330-008-0905-1

7. Hardcastle TC, Steyn E, Boffard K, et al. Guideline for the assessment of trauma centres in South Africa. S Afr Med J. 2011;101:189-194.

8. Department of Health. National Health Act (61:2003): Policy on national health insurance. Pretoria: Government Gazette No. 34523:657; 12 August 2011.

9. MRC-UNISA. A profile of fatal injuries in South Africa. Pretoria: Medical Research Council and Institute for Social and Health Sciences, University of South Africa; 2010.

10. Easton R, Sisak K, Balogh Z. Time to computed tomography scanning for major trauma patients: The Australian reality. ANZ J Surg. 2012; 82:644-647. http://dx. doi.org/10.1111/j.1445-2197.2012.06150.x

11. Rados A, Tiruta C, Xiao Z, et al. Does trauma team activation associate with the time to CT scan for those suspected of serious head injuries? World J Emerg Surg. 2013;8:48. http://dx.doi.org/10.1186/1749-7922-8-48

12. Hilbert P, Zur Nieden K, Hofmann, Hoeller I, Kock R, Stuttmann R. New aspect in the emergency room management of critically injured patients: A multi-slice CToriented care algorithm. Int J Care Injured. 2007;38:552-558. http://dx.doi. org/10.1016/j.injury.2006.12.023

13. Wurmb TE, Frühwald $P$, Hopfner W, et al. Whole-body multislice computed tomography as the first line diagnostic tool in patients with multiple injuries: The focus on time. J Trauma. 2009;66:658-665. http://dx.doi.org/10.1097/ TA.0b013e31817de3f4

14. Weinberg B, Richter M, Champine J, Morriss M, Browning T. Radiology resident preliminary reporting in an independent call environment: Multiyear assessment of volume, timeliness, and accuracy. J Am Coll Radiol. 2015;12:95-100. http:// dx.doi.org/10.1016/j.jacr.2014.08.005

15. Boland GWL, Halpern EF, Scott Gazelle G. Radiologist report turnaround time: Impact of pay-for-performance measures. AJR. 2010;195:707-711. http://dx.doi. org/10.2214/AJR.09.4164 